\title{
ALIMENTAÇÃO DE LEITÕES EM CRECHE COM DIETAS CONTENDO ÁCIDO ASCÓRBICO E BIOFLAVONÓIDES
}

\author{
FEEDING PIGLETS IN POST WEANING WITH DIETS CONTAINING \\ ASCORBIC ACID AND BIOFLAVONOIDS
}

\author{
Lehnen, C.R. ${ }^{1 *}$, Lovatto, P.A. ${ }^{1}$, Andretta, I. ${ }^{1}$, Rossi, C.A. ${ }^{1}$, Hauschild, L. ${ }^{1}$, Cavazini, N.C. ${ }^{1}$ \\ e Fraga, B.N. ${ }^{1}$
}

1Departamento de Zootecnia. Universidade Federal de Santa Maria. Santa Maria, RS. Brasil. *cheilalehnen@gmail.com

\section{PalaVRas ChaVE ADICIONAIS \\ Aditivos. Extratos cítricos. Vitamina C.}

\section{RESUMO}

Foi realizado um experimento para avaliar o desempenho de leitões em creche alimentados com dietas contendo ou não extratos cítricos. Foram utilizados 32 leitões, meio-irmãos paternos, com peso vivo médio inicial de $6,2 \mathrm{~kg}( \pm 0,87)$, durante 42 dias. $\mathrm{O}$ delineamento experimental foi o de blocos ao acaso, com dois tratamentos: dieta controle (DC) e DC + $750 \mathrm{mg} \mathrm{kg}^{-1}$ de um composto contendo extratos cítricos (ácido ascórbico + bioflavonóides), com oito repetições e dois animais por unidade experimental. O peso vivo final foi $4 \%$ superior $(p<0,01)$ nos leitões alimentados com dietas contendo extratos cítricos em relação aos animais do grupo controle (30,5 vs. $28,9 \mathrm{~kg})$. O consumo de ração dos animais alimentados com dietas contendo extratos cítricos foi $9 \%$ superior $(p<0,01)$ ao grupo controle $(0,77$ vs. $0,84 \mathrm{~kg})$. O ganho de peso foi $3,7 \%$ superior $(p=0,05)$ em leitões alimentados com dietas contendo extratos cítricos. A conversão alimentar não foi influenciada $(p>0,05)$ pela adição de extratos cítricos na dieta. $A$ adição de extratos cítricos contendo bioflavonóides e ácido ascórbico nas dietas pode melhorar o desempenho zootécnico de leitões pelo aumento na ingestão voluntária de alimento.

\section{SUMMARY}

An experiment was carried out to evaluate the post-weaning performance of piglets fed during 42 days diets with or without citric extracts. Thirty-two weanling piglets were used, with initial

Recibido: 20-9-10. Aceptado: 22-9-11.

\author{
AdDitionAl KEYWORDS \\ Additives. Citric extracts. Vitamin C.
}

average live weight of $6.2 \mathrm{~kg}( \pm 0.87)$, during one period of 42 days. A complete randomized block design was utilized, with two treatments: control diet $C D$, and $C D+750 \mathrm{mg} \mathrm{kg}^{-1}$ of citric extracts (ascorbic acid + bioflavonoids), with eight replicates and two animals per experimental unit. Final live weight was $4 \%$ higher $(p<0.01)$ in the animals fed with diets containing citric extracts than control group (30.5 vs. $28.9 \mathrm{~kg}$ ). Average daily feed intake of animals fed diets with citric extracts was $9 \%$ higher $(p<0.01)$ than control group ( $0.77 \mathrm{vs.} 0.84 \mathrm{~kg})$. Average daily weight gain was increased by $3.7 \%(p=0.05)$ in piglets fed diets containing citric extracts. The feed conversion ratio was not influenced $(p>0.05)$ by the citric extracts. Addition of citric extracts containing bioflavonoids and ascorbic acid in diets can improve piglet post-weaning performance by increasing voluntary feed intake.

\section{INTRODUÇÃO}

A busca por produtividade induziu importantes avanços na suinocultura, especialmente em nutrição. Neste contexto, o uso de antibióticos promotores de crescimento foi fundamental para a melhora na eficiência alimentar e no desempenho dos animais(Utiyama, 2004). Com a possibilidade da indução de resistência cruzada com patógenos humanos e a presença de resí- 
duos de antibióticos nos produtos finais (carne, leite e ovos), vários países têm restringido o uso de antimicrobianos como promotores de crescimento na alimentação animal (Soncini, 1999). Com isso, surgiram restrições e novas regulamentações quanto ao uso de antibióticos e quimioterápicos na alimentação animal, os quais atualmente são utilizados somente com finalidade curativa. A pressão pela retirada dos antimicrobianos das dietas tem aumentado a busca por produtos alternativos que garantam o máximo desempenho dos animais sem afetar a qualidade do produto final.

Em vista disso, alternativas têm sido pesquisadas e viabilizadas, dentre as quais o uso de extratos vegetais na dieta (Hauptli e Lovatto, 2006; Costa et al., 2007; Windisch et al., 2008). Esses aditivos têm ações antimicrobianas e imunomoduladoras sobre a microbiota intestinal e digestão dos nutrientes (Mellor, 2000). Os efeitos variam com a origem dos extratos e, portanto, com seus princípios ativos (Costa et al., 2007). Os principais componentes do extrato derivado de frutas cítricas são os compostos polifenólicos (bioflavonóides) e o ácido ascórbico.

Os bioflavonóides são compostos polifenólicos, encontrados em frutas, vegetais, sementes e flores, que apresentam estrutura química e características variadas. Esses compostos, pela ação inibitória sobre algumas enzimas e propriedade quelante a metais, inibem as reações em cadeia induzidas por radicais livres (Erlund, 2004). Dentre os bioflavonóides, os mais estudados são a quercetina, a miricetina, a rutina e a naringenina (Benavente-Garcia et al., 1997). São conhecidos como antioxidantes naturais e apresentam atividade antiinflamatória, antimicrobiana, antialergênica e imunoestimulante (Erlund, 2004; Cushnie e Lamb, 2005).

O ácido ascórbico é um importante cofator enzimático que participa de diversos processos de óxido-redução, de inativação de radicais livres e do aumento da absorção de ferro (Mahan et al., 2004). Ele também atua na colagênese e na síntese de epinefrina, corticosteroides e ácidos biliares (Aranha et al., 2000). Os benefícios da utilização terapêutica do ácido ascórbico, em estudos com animais, ainda incluem a proteção contra os efeitos colaterais de alguns medicamentos (Bianchi e Antunes, 1999).

Pelas propriedades individuais e ação sinérgica de seus princípios ativos, o uso de extratos cítricos nas dietas pode beneficiar o desempenho dos leitões em creche. No entanto, as informações disponíveis sobre a adição de extratos cítricos em dietas para suínos são incipientes (Gallois e Oswald, 2008). Este trabalho foi conduzido, portanto, com o objetivo de avaliar os efeitos do ácido ascórbico e dos bioflavonóides, através da adição de extratos cítricos em dietas para leitões em creche.

\section{MATERIAL E MÉTODOS}

Foram utilizados 32 leitões machos castrados homogêneos geneticamente, oriundos de criação comercial e desmamados aos 21 dias de idade, com peso de 6,2 $( \pm 0,87)$ quilogramas. Os leitões foram alojados em 16 baias elevadas $(1,50 \times 1,37 \times 0,80 \mathrm{~m}) \mathrm{com}$ piso parcialmente vazado, equipadas com comedouros semiautomáticos e bebedouros tipo concha. A temperatura da sala-creche foi mantida dentro da zona de conforto térmico recomendada para a fase. O delineamento experimental foi de blocos ao acaso com dois tratamentos (dieta controle e dieta controle $+750 \mathrm{mg} \mathrm{kg}^{-1}$ de Bioll ${ }^{\circledR}$ Herbotec $\left.^{1}\right)$. Cada tratamento teve oito repetições e dois animais por unidade experimental. O critério para a formação dos blocos foi o peso vivo inicial dos leitões. A dieta controle foi formulada para atender as recomendações

\footnotetext{
${ }^{1}$ Os principais componentes do Bioll ${ }^{\circledR}$ Herbotec são o ácido ascórbico e os bioflavonóides, compostos derivados de frutas cítricas como laranja (Citrus sinensis) e bergamota (Citrus aurantium bergamia), adicionadas a um veículo.
} 


\section{EXTRATOS CÍTRICOS NA ALIMENTAÇÃO DE LEITÕES}

Tabela I. Ingredientes e composição calculada da dieta controle ${ }^{1}$. (Ingredients and calculated composition of the control diet).

\begin{tabular}{|c|c|c|c|}
\hline Ingredientes & Fase 1 & Fase 2 & Fase 3 \\
\hline Milho & 31,91 & 43,85 & 52,87 \\
\hline Farelo de soja (48\% PB) & 23,30 & 34,75 & 36,75 \\
\hline Óleo de soja & 3,09 & 3,96 & 4,12 \\
\hline Fosfato bicálcico & 1,70 & 2,44 & 2,27 \\
\hline DL-metionina & 0,08 & 0,01 & - \\
\hline Óxido de zinco, mg kg-1 & 75 & 150 & 90 \\
\hline Colistina, $\mathrm{mg} \mathrm{kg}^{-1}$ & 52 & 31 & 36 \\
\hline Halquilol, $\mathrm{mg} \mathrm{kg}^{-1}$ & 120 & 61 & 96 \\
\hline Núcleo² & 40,00 & - & - \\
\hline Núcleo $^{3}$ & - & 15 & - \\
\hline Premix mineral e vítaminico ${ }^{4}$ & - & - & 4,00 \\
\hline \multicolumn{4}{|l|}{ Composição calculada ${ }^{5}$} \\
\hline Energia metabolizável, kcal/kg & 3265 & 3265 & 3265 \\
\hline Proteína bruta, \% & 22,50 & 20,90 & 20,40 \\
\hline Cálcio, \% & 0,77 & 0,71 & 0,67 \\
\hline Fósforo disponível, \% & 0,37 & 0,51 & 0,49 \\
\hline Lisina, \% & 1,12 & 1,04 & 1,12 \\
\hline Metionina, \% & 0,30 & 0,29 & 0,32 \\
\hline Metionina+cistina, \% & 0,63 & 0,58 & 0,63 \\
\hline Treonina & 0,70 & 0,74 & 0,79 \\
\hline Triptofano & 0,20 & 0,25 & 0,27 \\
\hline
\end{tabular}

${ }^{1}$ Matéria natural. ${ }^{2}$ Suplemento mineral proteico, vitamínico e energético para suínos produzido pela MIGPLUS, contendo por kg de produto: Vit. A (37500 UI), Vit. B1 (3,75 mg), Vit. B12 (100 mcg), Vit. D6 (5000 UI), Vit. E (300 mg), Vit. K3 (3,75 mg), Zn (187 mg), Ác. Fólico (3,37 mg), Ác. Pantotênico (30 mg), $\mathrm{Ca}(1,8 \%), \mathrm{P}(1 \%) .{ }^{3}$ Suplemento vitamínico e mineral, contendo por kg de produto: Ác. Fólico $(2 \mathrm{mg})$, Ác. Nicotínico (200 mg), Antioxidante (250 mg), Biotina (0,66 mg), Ca (50 g), Co (5 mg), Co (5 mg), Cu (100 $\mathrm{mg}$ ), Colina (2400 mg), EM/kg (3265), EE (5\%), Fe (750 mg), P (22 g), Lactose (27\%), Lisina (10000 mg), Metionina (6000 mg), Promotor de crescimento $(200 \mathrm{mg})$, Treonina (3000 mg), Vit. A (66500 UI), Vit. B1 (10 mg), Vit. B2 (36 mg), Vit. B6 (26 mg), Vit. B12 (133 mcg), Vit. D3 (13300 UI), Vit. E (500 mg), Vit. K3 $(18 \mathrm{mg}), \mathrm{Zn}(600 \mathrm{mg}) .{ }^{4}$ Suplemento vitamínico e mineral, contendo por $\mathrm{kg}$ de produto: Vit. A (170000 UI), Vit. B1 (17 mg), Vit. B2 (65 mg), Vit. B6 (34 mg), Vit. B12 (340 mg), Vit. D3 (34000 UI), Vit. E (500 mg), Vit. K3 (35 mg), Ác. Fólico (13 mg), Ác. Nicotinico (430 mg), Ác. Pantotênico (175 mg), Ca (220 g), P $(62 \mathrm{~g}), \mathrm{Na}(62 \mathrm{~g})$, promotor de crescimento $(1500 \mathrm{mg})$, Antioxidante $(250 \mathrm{mg}) .{ }^{5}$ Valores calculados com base nas exigências nutricionais para as fases segundo National Research Council (NRC, 1998).

nutricionais do NRC (1998), divididas em três fases: 1 a 14, 15 a 28 e 29 a 42 dias (tabela I). Em todas as fases foi mantido na dieta, o óxido de zinco e promotores de crescimento (colistina e halquilol). Os animais receberam alimentação à vontade e tiveram livre acesso à água. Os dados de ganho de peso foram obtidos por pesagens semanais individuais dos animais. O consumo diário de ração foi obtido pela pesagem da ração fornecida menos as sobras presentes nos comedouros. A conversão alimentar foi estimada a partir das variáveis anteriores.

Os dados foram analisados através do programa Statistical Analysis Systems (SAS, 2000) com o uso do procedimento 
Proc Mixed. Os efeitos incluídos no modelo analítico foram tratamentos $(\mathrm{T})$, período $(\mathrm{P})$ e interação entre tratamento e período $(\mathrm{T} *$ $\mathrm{P})$. Na análise de medidas repetidas no tempo foi utilizada a estrutura de covariância autorregressiva (Xavier, 2000; Malheiros, 2001).

\section{RESULTADOSEDISCUSSÃO}

Não houve interação $(p>0,05)$ entre tratamentos e período nos resultados de peso vivo dos leitões (tabela II). A adição de extratos cítricos na dieta aumentou $(p<0,01)$ em $4 \%$ o peso vivo final dos leitões em relação aos alimentados com a dieta controle. No período entre o desmame e o final de creche, os leitões sofrem com diversos fatores estressantes (Teodoro et al., 2008). Os radicais livres liberados em excesso durante o estresse podem resultar em lesões celulares, responsáveis por alterações fisiológicas predisponentes a processos patológicos. Embora o organismo consiga prevenir reações indesejáveis e reparar moléculas de tecidos danificados, em situações de estresse oxidativo esses mecanismos têm atividade reduzida, o que altera o metabolismo e algumas funções hepáticas (Ferreira e Matsubara, 1997). A quercetina contida nas frutas cítricas é responsável pelo aumento nas concentrações de glutationa no fígado, inibindo a formação de radicais livres e a peroxidação (Nijveldt et al., 2001). Além disso, a glutationa é um precursor da síntese do ácido ascórbico, que no organismo atua como um importante antioxidante. Assim, é provável que a presença dos bioflavonóides e do ácido ascórbico contidos no extrato de frutas cítricas tenha melhorado a condição metabólica do animal, através da atuação como imunoestimulante, antioxidante e hepatoprotetor (Decker, 1997; Mahan et al., 2004). Neste contexto, os resultados obtidos neste estudo, corroboram com os realizados por Mahan et al. (2004) que verificaram que dietas contendo até $900 \mathrm{mg} \mathrm{kg}^{-1}$ de ácido
Tabela II. Peso vivo de leitões alimentados com dietas com ou sem adição de extratos citricos. (Body weight of piglets fed diets with or without citric extracts addition).

\begin{tabular}{lcc}
\hline \multirow{2}{*}{ Semana } & \multicolumn{2}{c}{ Tratamentos } \\
& Controle & Extratos cítricos \\
\hline Peso inicial & 6,25 & 6,25 \\
2 & 7,29 & 7,54 \\
3 & 9,75 & 10,31 \\
4 & 13,66 & 14,75 \\
5 & 18,14 & 19,28 \\
6 & 23,47 & 24,22 \\
Peso final & 28,90 & 30,05 \\
& \multicolumn{2}{c}{0,17} \\
Epr & \multicolumn{2}{c}{$\mathrm{p}<0,01$} \\
Tratamento $(T)$ & \multicolumn{2}{c}{$\mathrm{p}<0,01$} \\
Período $(P)$ & \multicolumn{2}{c}{$\mathrm{p}=0,82$} \\
T*P & \multicolumn{2}{c}{} \\
\hline
\end{tabular}

Epr: erro padrão das médias.

ascórbico aumentam o peso vivo de leitões ao final da creche.

Os resultados de consumo de alimento, ganho de peso e conversão alimentar são apresentados na tabela III. Não houve interação $(p>0,05)$ entre tratamentos e período nas variáveis estudadas. Entretanto, a dieta contendo extratos cítricos aumentou $(\mathrm{p}<0,01)$ em média $9 \%$ o consumo de ração pelos leitões. Nas primeiras duas semanas o consumo de ração foi 11 e 12,6\% superior em leitões que receberam a dieta contendo extratos cítricos em relação aos animais da dieta controle. Nas demais semanas avaliadas a dieta contendo extratos cítricos aumentou ( $\mathrm{p}<0,01)$ em média $8 \%$ o consumo de ração pelos leitões. Os fenóis e alcaloides liberados pela quercetina, rutina e naringenina podem estimular o consumo de alimento (Benavente-Garcia et al., 1997; Ader et al., 2000). Além disso, estes compostos atuam como conservantes impedindo a oxidação dos lipídeos, o que mantêm preservada a qualidade nutricional e a palatabilidade das dietas (Windisch et al., 2008). 


\section{EXTRATOS CÍTRICOS NA ALIMENTAÇÃO DE LEITÕES}

Desse modo, a presença dos bioflavonóides e outros compostos fenólicos nos extratos cítricos podem melhorar a palatabilidade da dieta e aumentar o consumo de alimento pelos animais.

A presença de ácido ascórbico e bioflavonóides nos extratos cítricos adicionados à dieta aumentaram $(\mathrm{p}=0,05)$ em $3,7 \%$ o ganho de peso dos leitões. No entanto, a conversão alimentar dos animais alimentados com dietas contendo extratos cítricos e do grupo controle não foi alterado $(1,40 \mathrm{vs}$. $1,42 ; p>0,05)$. Dessa forma, os resultados indicam que o aumento do consumo através da adição de extratos cítricos na dieta refletiu em um aumento proporcional no ganho de peso dos animais. Neste contexto, a adição de extratos cítricos pode influenciar sob dois aspectos, o primeiro, relacionado à liberação de compostos fenólicos nas dietas e o segundo, associado ao efeito sinérgico entre o ácido ascórbico e os bioflavonóides sobre os processos de digestão e utilização dos nutrientes (Windisch et al., 2008). Resultados semelhantes foram obtidos com dietas contendo $150 \mathrm{mg} \mathrm{kg}^{-1}$ de ácido ascórbico por Fernandéz-Dueñas et al. (2008).

No entanto, a conversão alimentar dos animais alimentados com dietas contendo extratos cítricos e do grupo controle não foi alterado $(1,40 v s .1,42 ; \mathrm{p}>0,05)$. Neste sentido, a ação digestiva e metabólica dos bioflavonóides pode não ter sido suficiente para alterar significativamente a conversão alimentar. Além disso, outro aspecto a ser considerado é a presença de outros promotores de crescimento como a colistina, halquilol e óxido de zinco que influenciaram no desempenho dos animais do tratamento controle. Contudo é importante salientar que a adição de extratos cítricos estimulou o consumo de alimento nas primeiras semanas na fase de creche, sendo esta fase considerada crítica para a utilização digestiva dos nutrientes.

Entre outros benefícios da utilização de bioflavonóides e ácido ascórbico está a proteção de células e tecidos de lesões induzidas por radicais livres e por toxinas liberadas pelos micro-organismos aderidos ao epitélio intestinal (Cushnie e Lamb, 2005). Assim, em nível sistêmico, os extratos cítricos podem otimizar as defesas do organis-

Tabela III. Desempenho de leitões alimentados com dietas com ou sem adição de extratos citricos $(E C)$. (Performance of piglets fed diets with or without citric extracts (EC) addition).

\begin{tabular}{|c|c|c|c|c|c|c|}
\hline \multirow[b]{2}{*}{ Semana } & \multicolumn{2}{|c|}{ Consumo de alimento (kg/dia) } & \multicolumn{2}{|c|}{ Ganho médio de peso (kg/dia) } & \multicolumn{2}{|c|}{ Conversão alimentar } \\
\hline & Controle & EC & Controle & EC & Controle & EC \\
\hline 1 & 0,16 & 0,18 & 0,15 & 0,18 & 1,29 & 1,04 \\
\hline 2 & 0,76 & 0,87 & 0,35 & 0,39 & 1,10 & 1,12 \\
\hline 3 & 0,67 & 0,75 & 0,56 & 0,63 & 1,23 & 1,19 \\
\hline 4 & 0,90 & 0,98 & 0,64 & 0,65 & 1,41 & 1,52 \\
\hline 5 & 1,13 & 1,21 & 0,71 & 0,70 & 1,58 & 1,70 \\
\hline 6 & 1,37 & 1,49 & 0,83 & 0,83 & 1,89 & 1,79 \\
\hline $1-6$ & 0,77 & 0,84 & 0,54 & 0,56 & 1,42 & 1,40 \\
\hline Epr & \multicolumn{2}{|c|}{0,01} & \multicolumn{2}{|c|}{0,01} & \multicolumn{2}{|c|}{0,03} \\
\hline Tratamento $(\mathrm{T})$ & \multicolumn{2}{|c|}{$p<0,01$} & \multicolumn{2}{|c|}{$p=0,05$} & \multicolumn{2}{|c|}{$p=0,61$} \\
\hline Período $(\mathrm{P})$ & \multicolumn{2}{|c|}{$p<0,01$} & \multicolumn{2}{|c|}{$p<0,01$} & \multicolumn{2}{|c|}{$p<0,01$} \\
\hline $\mathrm{T}^{*} \mathrm{P}$ & \multicolumn{2}{|c|}{$p=0,52$} & \multicolumn{2}{|c|}{$p=0,63$} & \multicolumn{2}{|c|}{$p=0,14$} \\
\hline
\end{tabular}

Epr: erro padrão das médias. 


\section{LEHNEN, LOVATTO, ANDRETTA, ROSSI, HAUSCHILD, CAVAZINI E FRAGA}

mo (Mahan et al., 2004). Além de melhorar a utilização dos nutrientes das dietas, através da redução de substrato disponível a bactérias patogênicas, reduzindo a incidência de diarréias em leitões (Kamel, 2001). Entretanto, a maioria dos estudos com bioflavonóides apresentam respostas benéficas em nível celular. Embora a utilização de extratos cítricos na dieta de leitões tenha apresentado resultados promissores, são necessários maiores estudos envolvendo o desempenho zootécnico e a utilização digestiva e metabólica destes compostos fenólicos em suínos.

\section{CONCLUSÕES}

A adição de $750 \mathrm{mg} \mathrm{kg}^{-1}$ de extratos cítricos, contendo bioflavonóides e ácido ascórbico, aumenta o consumo de ração, o

\section{BIBLIOGRAFIA}

Ader, P., Wessmann, A. and Wolffram, S. 2000. Bioavailability and metabolism of the flavonol quercetin in the pig. Free Radical Bio Med, 28: 1056-1067.

Aranha, F.Q., Barros, Z.F., Moura, L.S., Gonçalves, M.C.R., Barros, J.C., Metri, J.C. and Souza, M.S. 2000. The role of vitamin $C$ in organic changes in aged people. Rev Nutr, 13: 89-97.

Benavente-Garcia, O., Castillo, J., Marin, F.R., Ortunõ, A. and Del Río, J.A. 1997. Uses and properties of citrus flavonoids. J Agric Food Chem, 45: 4505-4515.

Bianchi, M.D.L.P. and Antunes, L.M.G. 1999. Free radicals and the main dietary antioxidants. $R e v$ Nutr, 12: 123-130.

Costa, L.B., Tse, M.L.P. e Miyada, V.S. 2007. Extratos vegetais como alternativas aos antimicrobianos promotores de crescimento para leitões recém-desmamados. Rev Bras Zootecn, 36: 589-595.

Cushnie, T.P. and Lamb, A.J. 2005. Antimicrobial activity of flavonoids. Int $\mathrm{J}$ Antimicrob $\mathrm{Ag}, 26$ 343-356.

Decker, E.A. 1997. Phenolics: prooxidants or antioxidants? Nutr Rev, 55: 396-398.

Erlund, I. 2004. Review of the flavonoids quercetin, ganho médio diário e o peso vivo de leitões em final de creche. A adição de extratos cítricos contendo bioflavonóides e ácido ascórbico nas dietas pode melhorar o desempenho zootécnico de leitões pelo aumento na ingestão voluntária de alimento.

\section{AGRADECIMENTOS}

Os autores agradecem à Herbotec do Brasil Nutrição Animal Ltda pelo apoio na realização do experimento. À Comissão de Aperfeiçoamento de Pessoal de Nível Superior (CAPES) pelas bolsas de mestrado de Ines Andretta e Bruno Neutzling Fraga e de doutorado de Cheila Roberta Lehnen. Ao Conselho Nacional para o Desenvolvimento Científico e Tecnológico $(\mathrm{CNPq})$ pela bolsa de Produtividade em Pesquisa de Paulo Alberto Lovatto. hesperetin, and naringenin. Dietary sources, bioactivities, bioavailability and epidemiology. Nutr Res, 24: 851-874.

Fernández-Dueñas, D.M., Mariscal, G., Ramírez, E. and Cuarón, J.A. 2008. Vitamin C and [beta]carotene in diets for pigs at weaning. Anim Feed Sci Technol, 146: 313-326.

Ferreira, A.L.A. e Matsubara, L.S. 1997. Radicais livres: conceitos, doenças relacionadas, sistema de defesa e estresse oxidativo. Rev Ass Med Bras, 43: 61-68.

Gallois, M. and Oswald, I.P. 2008. Immunomodulators as efficient alternatives to in-feed antimicrobials in pig production? Archiva Zootechnica, 11: 15-32.

Hauptli, L. e Lovatto, P.A. 2006. Alimentação de porcas gestantes e lactantes com dietas contendo saponinas. Cienc Rural, 36: 610-616.

Kamel, C. 2001. Tracing modes of action and roles of plant extracts in non-ruminants. In: Garnsworthy, P.C. and Wiseman (Eds.). Recent advances in animal nutrition. Nottingham University. Nottingham, UK. pp. 135-150.

Mahan, D.C., Ching, S. and Dabrowski, K. 2004. Developmental aspects and factors influencing the synthesis and status of ascorbic acid in the

Archivos de zootecnia vol. 61, núm. 233, p. 108. 


\section{EXTRATOS CÍTRICOS NA ALIMENTAÇÃO DE LEITÕES}

pig. Annu Rev Nutr, 24: 79-103.

Malheiros, E.B. 2001. Precisão da análise de experimentos com medidas repetidas no tempo usando procedimentos do SAS. Rev Mat Estat, 19: $253-272$.

Mellor, S. 2000. Alternatives to antibiotic. Pig Progr, 16: 18-21.

Nijveldt, R.J., Van Nood, E., Van Hoorn, D.E., Boelens, P.G., Van Norren, K. and Van Leeuwen, P.A.M. 2001. Flavonoids: a review of probable mechanisms of action and potential applications. Annu Rev Nutr, 74: 418-425.

NRC. 1998. National Research Council. Nutrient Requirement of Swine. $10^{\text {th }}$ revised edition.

SAS. 2000. Statistical Analysis System. System for Windows, release 8.0. Cary, NC. 01 CDROM.

Soncini, R.A. 1999. Restrições do uso de aditivos na alimentação animal: expectativa da agroindústria. Em: Simpósio sobre as implicações socioeconômicas do uso de aditivos na produção animal. Piracicaba. São Paulo. pp. 99-104.
Teodoro, S.M., Berto, D.A., Padovani, C.R., Chaves, M.A. e Panizza, J.C. 2008. Leitões lactentes e desmamados alimentados com dietas farelada ou extrusada seca e úmida. Arch Zootec, 57: 549-552.

Utiyama, C.E. 2004. Utilização de agentes antimicrobianos, probióticos, prebióticos e extratos vegetais como promotores do crescimento de leitões recém-desmamados. Tese (Doutorado em Agronomia). Escola Superior de Agricultura Luiz de Queiroz. Piracicaba, São Paulo. 110 pp.

Windisch, W., Schedle, K., Plitzner, C. and Kroismayr, A. 2008. Use of phytogenic products as feed additives for swine and poultry. $J$ Anim Sci, 86: E140-E148.

Xavier, L.H. 2000. Modelos univariado e multivariado para analise de medidas repetidas e verificação da acuracia do modelo univariado por meio de simulação. Dissertação (Mestrado em Estatística e Experimentação Agronômica). Escola Superior Luiz de Queiroz. Piracicaba. 91 pp. 